\section{The Brazilian Society of Pediatrics, Department of Infectious Diseases guidelines for the management of children and adolescents with acute pharyngitis}

Dear Editor,

In response to the study published by dos Santos \& Berezin ${ }^{1}$ and the editorial published by Ejzenberg, ${ }^{2}$ we would like to comment on the importance of selecting which patients should have the rapid testing or oropharynx cultures performed.

Sore throat is one of the most common causes of seeking medical care. ${ }^{3}$ One of the causes of this complaint is acute pharyngitis (AP). ${ }^{3}$ Although the great majority of these patients have viral infections, it is of fundamental importance to identify which patients have AP secondary to infections by Streptococcus pyogenes. ${ }^{4}$ Viral AP has a benign prognosis without complications or sequelae and requires no specific treatment whereas streptococcal AP can progress with suppurative complications, such as periamygdalar abscesses, or non-suppurative complications such as rheumatic fever, which can be prevented with appropriate antimicrobial treatment of the primary condition (streptococcal AP). ${ }^{3}$

Differential diagnosis is based on clinical assessment: involvement of other mucosas such as conjunctiva or nasal mucosa with coryza, and also hoarseness or wheezing are uncommon to streptococcal AP, but frequent with viral AP. 5 Exudate may be present in either case. There is also significant difference in the age of the affected groups: infection by $S$. pyogenes predominantly affects individuals aged 5 to 15 years, and it is rare in children under 2 years old whereas viral AP can occur at any age and predominates in frequency among infants. ${ }^{5}$

Typically, streptococcal AP has sudden onset with intense sorethroat, fever, headache, nausea, vomiting and abdominal pain, and the pharynx may be inflamed, with swollen tonsils and edematous uvula; cervical lymph nodes are habitually enlarged and painful. Nevertheless, no one element of the clinical history or the physical examination alone is sensitive and specific enough to rule out or diagnose streptococcal etiology. 3

In virtue of the high frequency of $\mathrm{AP}$, the benefits of antimicrobial treatment for patients whose etiology is streptococcal, the lack of benefit from the use of antimicrobials when etiology is viral and the greater frequency of viral agents of AP (70-85\%), it has become necessary to increase the precision of its etiologic diagnosis in order to make the use of antimicrobials more accurate. The gold standard for the diagnosis of streptococcal etiology is oropharynx culture. Material should be collected by swab from the amygdala and the posterior pharynx wall with the objective of maximizing the accuracy of results. ${ }^{3}$ There is, however, a latent period of 24 to 48 hours between collecting the material and the culture results becoming available. With the objective of obtaining a laboratory result during the initial consultation, a quick test was developed (rapid antigen detection testing), the specificity and sensitivity of which has been observed at $95 \%$ and $80-$ $90 \%$, respectively. ${ }^{4}$ It is important to point out that the rapid test's accuracy is dependent on the selection of which patients are tested, i.e. the more compatible with streptococcal etiology the presentation is (sudden onset, fever, painful throat, with no conjunctivitis, coryza, coughing, hoarseness, prior stomatitis or diarrhea), the greater the test's accuracy. 4 The exclusion of patients with signs of viral respiratory infection (rhinorrhea, conjunctivitis, coughing and/or sneezing) by Santos \& Berezin, ${ }^{1}$ without doubt influenced their results, since this exclusion increases the pre-test prevalence of streptococcal AP. This is the same method that is recommended for clinical practice, ${ }^{6}$ in contrast to what Ejzenberg ${ }^{2}$ has recommended, "to use the rapid test in all patients with acute pharyngitis."

Summing up, laboratory workup should proceed as follows: patients with clinical and epidemiological history compatible with streptococcal infection should be tested with the rapid test or have a culture taken; if the rapid test is positive antibiotics are indicated; if the rapid test is negative a culture is performed; in case of a positive culture antibiotic therapy is indicated and when the culture is negative treatment of symptoms is indicated (Figure 1 ).

The primary objective of antibiotic therapy is to eradicate streptococcus from the oropharynx and consequently prevent complications; another objective is to shorten the length of the illness. ${ }^{5}$ To date, no reports exist of S. pyogenes resistant to penicillin benzathine, which continues to be an effective treatment for streptococcal AP. ${ }^{5}$ Oral treatment options are penicillin $\mathrm{V}$ and amoxycillin, with the latter being preferred because of price and flavor. Amoxycillin $50 \mathrm{mg} / \mathrm{kg} /$ day twice daily (every $12 \mathrm{~h}$ ) has been demonstrated to be equivalent in clinical and bacteriological efficacy. This regimen is advantageous because of the convenient posology. ${ }^{7}$

For patients who are allergic to penicillin, but without immediate hypersensitivity, the first generation cephalosporins erythromycin and clindamycin are treatment options, however, cephalosporins are contraindicated for allergic patients with immediate hypersensitivity. ${ }^{8}$ Erythromycin can be used, specifically for the treatment of streptococcal AP in children, at doses of $40 \mathrm{mg} / \mathrm{kg} /$ day in two parts at 12 hour intervals. ${ }^{8}$ 


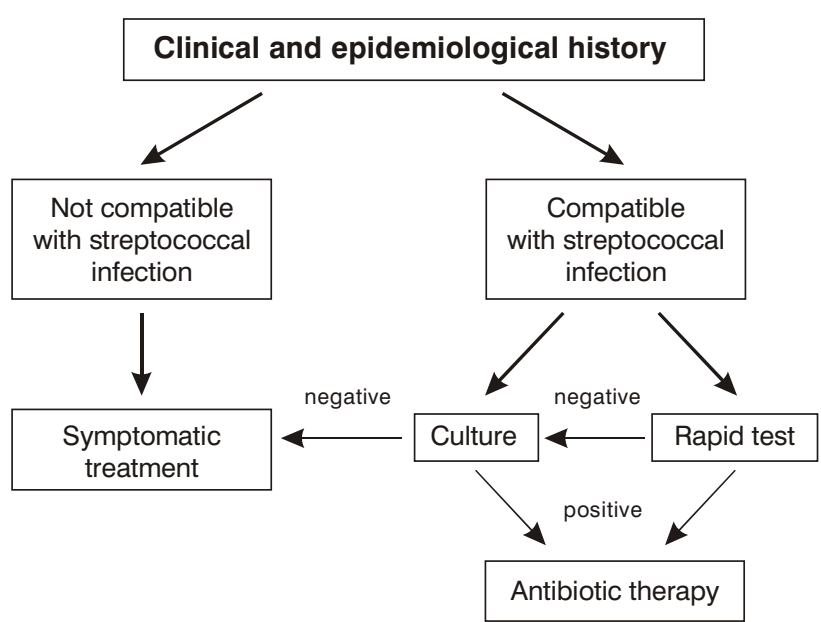

Figure 1 - Algorithm for the diagnosis and treatment of children and adolescents with acute pharyngitis

All of the oral antibiotics cited above should be taken for 10 days, irrespective of whether there is resolution of the clinical condition, since streptococcus is only eradicated after this length of antibiotic therapy. It is for this reason that very often, when compliance with treatment is doubtful, penicillin benzathine is the best option, given in a single intramuscular dose of $600,000 \mathrm{UI}$ for patients weighing less than or equal to $27 \mathrm{~kg}$ and 1,200,000 UI for those over $27 \mathrm{~kg} .8$

Strains of $S$. pyogenes resistant to macrolides have been documented in several different geographical areas. ${ }^{8}$ This signifies that one can expect less efficacy from these antimicrobials than from penicillins. Sulfonamides and tetracyclines are contraindicated for streptococcal AP because, although they contribute to resolving the clinical condition, they exhibit high rates of failure to eradicate $S$. pyogenes bacteriologically. 8

It is interesting to note that the efficacy of antibiotic therapy is much lower for the eradication of $S$. pyogenes in carriers. ${ }^{8}$ For this reason, antibiotic therapy is only indicated for carriers in the following situations: closed communities (orphanages for example), living with a household member who has rheumatic fever. ${ }^{8}$

\section{Acknowledgements}

Núcleo Gerencial of the Department of Infectious Diseases of the Brazilian Society of Pediatrics, for reviewing this manuscript.

\section{References}

1. dos Santos AG, Berezin EN. Comparação entre métodos clínicos e laboratoriais no diagnóstico das faringotonsilites estreptocócicas. J Pediatr (Rio J). 2005;81:23-8.

2. Ejzenberg B. A conduta frente ao paciente com faringite aguda. J Pediatr (Rio J). 2005;81:1-2.
3. Vincent MT, Celestin N, Hussain AN. Pharyngitis. Am Fam Physician. 2004;69:1465-70.

4. Gieseker $\mathrm{KE}$, Roe $\mathrm{MH}$, Mackenzie $\mathrm{T}$, Tood JK. Evaluating the American Academy of Pediatrics diagnostic standard for Streptococcus pyogenes pharyngitis: backup culture versus repeat rapid antigen testing. Pediatrics. 2003;111:e666-70.

5. Crump J, Harrison V, Shope T, Rion R. Pharyngitis. Guidelines for clinical care. UMMC Pharyngitis Guideline, December 2000. http://cme.med.umich.edu/pdf/guideline/phrngts.pdf. Accessed: 11/10/2005.

6. Bisno AL, Gerber MA, Gwaltney Jr JM, Kaplan EL, Schwartz RH. Practice guidelines for the diagnosis and management of group A streptococcal pharyngitis. Clin Infect Dis. 2002;35:113-25.

7. Cohen R, Levy C, Doit C, Rocque F, Boucherat M, Fitoussi F, et al. E. Six-day amoxicillin vs. ten-day penicillin $\vee$ therapy for group A streptococcal tonsillopharyngitis. Pediatr Infect Dis. 1996; $15: 678-82$

8. Pickering LK. RED BOOK: 2003 Report of the Committee on Infectious Diseases. 26th ed. Elk Grove Village, IL: American Academy of Pediatrics; 2003. p. 578-80.

\section{Cristiana M. Nascimento-Carvalho}

Doutora. Professora adjunta, Departamento de Pediatria, Faculdade de Medicina, Universidade Federal da Bahia (UFBA), Salvador, BA. E-mail: nascimentocarvalho@hotmail.com

\section{Heloísa H. Sousa Marques}

Doutora. Departamento de Pediatria, Faculdade de Medicina, Universidade de São Paulo (USP). Médica assistente, Instituto da Criança, Hospital das Clínicas, Faculdade de Medicina, USP, São Paulo, SP. E-mail: heloisahsm@icr.hcnet.usp.br

\section{Author's reply}

\section{Standardization of the management of patients with acute pharyngitis}

It was with satisfaction that I received the proposals made by Nascimento-Carvalho \& Marques ${ }^{1}$ for the treatment of acute pharyngitis, based on recommendations by Bisno et al.2,3 The standardization of conduct could start with the definition of acute pharyngitis, since there is not a single definition, surprising as that may be. This led the authors to define similar recommendations as different. On the other hand, the role of bacterial cultures in the etiologic diagnosis of acute pharyngitis, referred to by the authors, merits profound debate before recommendations are made to pediatricians, especially in developing countries.

\section{Definitions of acute pharyngitis}

Pulmonology and pediatrics textbooks recognize two common diseases of the upper airways - the common cold (nasopharyngitis or rhinitis) and acute pharyngitis/tonsillitis/ pharyngotonsillitis. ${ }^{4,5}$ The common cold causes coryza, nasal obstruction, sneezing, lacrimation, coughing little fever, with greatest incidence from 0 to 3 years. In acute pharyngitis 
there is fever and localized signs of inflammation, with incidence among toddlers and schoolchildren. The international code for the classification of diseases recognizes these two nosological entities. ${ }^{6}$ Nasopharyngitis was excluded from the acute pharyngitis study published in the Jornal de Pediatria, ${ }^{7}$ and I have did the same in the editorial. ${ }^{2}$

In the letter above, in the article by Bisno et al., ${ }^{3}$ in common with others, ${ }^{8}$ acute pharyngitis is not defined, however, it is clear from the text that the denomination includes nasopharyngitis. There does not appear to be any advantage in the clinical merger of two diseases, the simple cold of viral etiology and symptomatic treatment with the acute pharyngitis, whose cause and treatment must be established case by case. This diagnostic unification also makes the standardization of conduct difficult, as can be observed in the flowchart in Bisno et al. ${ }^{3}$ recommended by the authors of the letter. Observe that, according to the chart, having classified a patient as suffering from acute pharyngitis, a physician should evaluate whether the case is suggestive of streptococcal infection, to decide whether or not to request supplementary tests. In the original text, ${ }^{3}$ there is a supplementary table to aid in this decision, covering 15 parameters - 11 suggestive of streptococcal etiology and four of viral infection (conjunctivitis, coryza, coughing and diarrhea). This makes it difficult to employ the guidelines in pediatric practice, its prime objective; it would appear simpler to maintain the two diseases as distinct entities. In cases of doubts over whether to diagnose a cold or pharyngitis, prudence dictates considering the case of acute pharyngitis and investigating the etiology. The initial clinical diagnosis of acute pharyngitis should be re-evaluated throughout the course of the illness because of the possible occurrence of concomitant infections in the paranasal cavities, lungs and other sites in the organism. ${ }^{2}$

\section{Management of acute pharyngitis}

Management of the child with acute pharyngitis is based on consensus: 1) viral causes predominate and $S$. pyogenes causes 15 to $30 \%$ of cases; 2 ) there are no clinical features that can define etiology with certainty; 3) a proportion of pharyngitis cases caused by $S$. pyogenes are not recognized and go untreated; 4) clinical doubts about etiology lead pediatricians to prescribe antibiotics to half of all patients; 5 ) inappropriate prescriptions of antibiotics take place in 30 to $40 \%$ of cases. $2,3,8$ These factors have been observed in children from 2 years of age onwards, ${ }^{7}$ and support the recommendation made in the editorial for routine streptococcal testing in acute pharyngitis. $2,3,7,8$

\section{Rapid testing and cultures}

Rapid tests for streptococcal antigens have an increasing role in the etiological investigation of acute pharyngitis. $3,8,9$ This is because it offers immediate and precise results and can be performed by a clinician or a trained auxiliary, anywhere at anytime, with no special equipment and at low cost. The best versions have sensitivity rates over $90 \%$ and specificity at $95 \%$, approaching cultures, which achieve $96 \%$ in these evaluations. $3,8,9$ Bacterial cultures are the microbiological standard, ${ }^{3}$ but their role in clinical practice has recently been questioned. ${ }^{9}$ This is because of difficulties in obtaining, transporting and preserving adequately the culture media, and the fact that they must be performed in a specific location by a laboratory technician. The incubation period causes significant problems, as it delays the medical decision on whether to prescribe antibiotics and means the family must return to the hospital, which may cause some loss. Such difficulties mean that pediatricians rarely request cultures, in Brazil.

There is another factor that makes it difficult to include bacterial cultures in the management of acute pharyngitis. They are to be requested in cases of clinical evidence of streptococcal infection in the event of a negative rapid test result. There is only one parameter of clinical evidence that is specific to infection by $S$. pyogenes, - scarlatiniform exanthema. ${ }^{9}$ Such appropriate indications for a culture would occur very occasionally. ${ }^{7-9}$ Therefore it is other signs and symptoms - petechiae in mucosa, painful submandibular limphonodes, pharingeal exudate and high or persistent fever, that are used as evidence of streptococcal infection. 3,7,9 These signs and symptoms are common and have little specificity. $3,7,9$ The study carried out by Santos \& Berezin, ${ }^{7}$ in common with others, ${ }^{3}$ found that clinical evidence could justify antibiotics in $47 \%$ of cases, but only $21 \%$ of the patients had a positive rapid test result. If the recommendations made by Bisno et al. $^{3}$ are followed, we should be taking pharyngeal cultures in around one quarter of all pharyngitis cases, with a total increase of $2 \%$ in cases of streptococcus detected.

For all of the reasons above, faced with a clinical suspicion of infection by $S$. pyogenes, but a negative rapid test result, some authors have proposed that the rapid test should be repeated without recourse to bacterial cultures. ${ }^{9}$

\section{Conclusions and recommendations}

The assessment of acute pharyngitis must be clinical and etiological, the second being carried out by testing for $S$. pyogenes. Rapid tests for the detection of bacteria antigens have achieved sufficient performance for clinical use, whereas bacterial culture, the microbiological reference standard, involves several practical difficulties.

The establishment of recommendations on acute pharyngitis management, by medical societies, is in an opportune phase of development and pediatric infectious disease specialists have already made a contribution. However, in the attempt to improve and standardize recommendations, it would be interesting to include the expertise accumulated by other specialties - otorhinolaryngology, pneumology, emergency medicine and ambulatory pediatrics. The viability of recommendations could also benefit from assessments by epidemiologists and public health managers. At the end of the task, it is possible that we could have a Recommendation for Acute Pharyngitis circulated by the Brazilian Society of Pediatrics that serves as guidance in individual cases and also for public health planning.

\section{References}

1. Nascimento-Carvalho CM, Marques HH. Recomendação do Departamento de Infectologia da Sociedade Brasileira de Pediatria para conduta de crianças e adolescentes com faringoamigdalites agudas. J Pediatr (Rio J). 2005;82:79-80. 
2. Ejzenberg B. A conduta frente ao paciente com faringite aguda. J Pediatr (Rio J). 2005;81:1-2.

3. Bisno AL, Gerber MA, Gwaltney Jr JM, Kaplan EL, Schwartz RH. Practice guidelines for the diagnosis and management of group A streptococcal pharyngitis. Clin Infect Dis. 2002;35:113-26.

4. Asher MI. Infections of the upper respiratory tract. In: Lynn M, Taussig $L$, editors. Pediatric respiratory medicine. St. Louis: Mosby; 1999. p. 530-47.

5. Turner RB, Hayden GF. The common cold. In: Behrman RE, Kliegman R, Arvin AM, editors. Nelson textbook of pediatrics. 17th ed. Philadelphia: W. B. Saunders Co.; 2004. p. 1389-91.

6. Organização Mundial da Saúde. Classificação estatística internacional de doenças e problemas relacionados à saúde. CID-10. São Paulo: EDUSP; 2002.

7. dos Santos AG, Berezin I. Comparação entre métodos clínicos e laboratoriais no diagnóstico das faringotonsilites estreptocócicas. J Pediatr (Rio J). 2005;81:23-8.

8. Vincent MT, Celestin N, Hussain NA. Pharyngitis. Am Fam Physician. 2004;6:1465-71.

9. Gieseker KE, Roe MH, MacKenzie T, Todd JK. Evaluating the American Academy of Pediatrics diagnostic standard for Streptococcus pyogenes pharyngitis: backup culture versus repeat rapid antigen testing. Pediatrics. 2003;111:e666-70.

\section{Bernardo Ejzenberg}

Livre-docente. Professor, Instituto da Criança, Hospital das Clínicas, Faculdade de Medicina, Universidade de São Paulo (USP) e Divisão de Pediatria, Hospital Universitário, USP, São Paulo, SP.

\section{Authors' reply}

To the Editor of the Jornal de Pediatria,

We are grateful for the mention given our article $^{1}$ and are glad to take this opportunity to respond to the issues raised.

Not even a combination of signs and symptoms is capable of differentiating viral pharyngitis s from bacterial pharyngitis $s$ with certainty. This is the reason for which many authorities recommend that streptococcal pharyngitis (ST) in patients under clinical and epidemiological suspicion be diagnosed by means of microbiological tests. Currently bacterial resistance is growing alarmingly and it is necessary to reduce unnecessary antibiotic prescriptions. ${ }^{2}$

Both the sensitivity and specificity of the time-honored signs and symptoms used for diagnosing ST are poor. Steinhoff et al. ${ }^{3}$ carried out a study in Egypt and found that the finding of purulent exudate offered a sensitivity of just $31 \%$ and a specificity of $81 \%$ for the diagnosis of streptococcal pharyngitis. This finding was reproduced in our study in which just $50 \%$ of the patients with ST exhibited purulent exudate. This fact makes us reflect that, in addition to the problem of excessive unnecessary treatment, there is also the possibility that treatment will not be given when streptococcal pharyngitis is present, increasing the risk of serious non-suppurative sequelae.

The other option, of treating all children who present with fever and pharyngitis would lead to inappropriate treatment being given to a very large number of patients.

The exclusion criteria that we employed in our study lead to a reduction in the inappropriate use of antimicrobials. It is true though that one of our exclusion criteria, age less than three years, was not effective because patients aged two years are diagnosed. However, below three years of age, the suppurative complications of ST are extremely rarely observed.

The use of laboratory methods can be an aid for defining the disease, but criteria are needed to decide which patients should be tested. Patients over 3 years old with fever and pharyngitis in association with one further sign such as swollen glands, purulent exudate, odynophagia or abdominal pains associated with no coughing or running nose, should preferably be tested. A history of recent contact with a patient with streptococcal pharyngitis can also help diagnosis. ${ }^{2}$

\section{References}

1. dos Santos AG, Berezin EN. Comparação entre métodos clínicos e laboratoriais no diagnóstico das faringotonsilites estreptocócicas. J Pediatr (Rio J). 2005;81:23-8.

2. Swartz B, Marcy M, Philips WR, Gerber MA, Dowell SF. Pharyngitis - Principles of Judicious use of Antimicrobial agents. Pediatrics. 1998;101:171-4.

3. Steinhoff MC, Abd El Khalek MK, Khallaf N, Hamza HS, El Ayadi $A$, Orabi $A$, et al. Effectiveness of clinical guidelines for the presumptive treatment of streptococcal pharyngitis in Egyptian children. Lancet. 1997;350:918-21.

\section{Ana Gabriela Pires do Santos}

Mestre. Médica segundo assistente, Departamento de Pediatria, Santa Casa de Misericórdia de São Paulo, SP.

\section{Eitan Naaman Berezin}

Doutor. Chefe do Serviço de Infectologia Pediátrica, Santa Casa de Misericórdia de São Paulo, SP. 